\title{
3-point functions from twisted mass lattice QCD at small quark masses
}

\author{
Stefano Capitani \\ Institut für Physik/Theoretische Physik \\ Universität Graz, A-8010 Graz, Austria \\ E-mail: stefano.capitani@uni-graz.at
}

\section{Karl Jansen*, Mauro Papinutto, Andrea Shindler, Ines Wetzorke}

John von Neumann-Institut für Computing NIC,

DESY, Platanenallee 6, D-15738 Zeuthen, Germany

E-mail:

Karl.Jansen, Mauro.Papinutto, Andrea.Shindler, Ines.Wetzorke@desy.de

\section{Carsten Urbach}

Institut für Theoretische Physik, Freie Universität Berlin

Arnimallee 14, D-14195 Berlin, Germany

E-mail: Carsten.Urbach@physik.fu-berlin.de

We show at the example of the matrix element between pion states of a twist-2, non-singlet operator that Wilson twisted mass fermions allow to compute this phenomenologically relevant quantitiy at small pseudo scalar masses of $\mathrm{O}(270 \mathrm{MeV})$. In the quenched approximation, we investigate the scaling behaviour of this observable that is derived from a 3-point function by applying two definitions of the critical mass and find a scaling compatible with the expected $\mathrm{O}\left(a^{2}\right)$ behaviour in both cases. A combined continuum extrapolations allows to obtain reliable results at small pion masses, which previously could not be explored by lattice QCD simulations.

XXIIIrd International Symposium on Lattice Field Theory

25-30 July 2005

Trinity College, Dublin, Ireland

\footnotetext{
* Speaker.
} 


\section{Introduction}

Precision experiments allow to determine the parton distribution functions $f\left(x, Q_{0}^{2}\right)$ as a function of the momentum fraction $x$ and the energy scale $Q_{0}^{2}$. At a fi xed value of $Q_{0}^{2}$, this function is usually parametrized as $f(x) \propto x^{\alpha}(1-x)^{\beta}$ with exponents $\alpha, \beta$ that are obtained from global fi ts of the world-wide available experimental data (see ref. [1] for a general introduction). The bridge to results from non-perturbative calculations in lattice QCD are the moments $\int d x x^{n-1} f(x)$ of the parton distribution functions which are directly computable with lattice technues. From the phenomenological side, there has been a lot of work to estimate the errors for these moments [2, 3, , These investigations also demonstrated clearly that the uncertainties of the parton distribution functions can easily reach $100 \%$, in particular for the gluon distribution function. Hence, results from lattice calculations are highly welcome for phenomenologists and experimentalists.

Lattice calculations for moments of parton distribution functions have progressed very much over the last years. We control the continuum limit [5] and the fi nite size effects [6], we understand fully the non-perturbative, scale dependent renormalization [7] of the operators needed to compute the moments and we can obtain matrix elements of such operators with a good precision. Nevertheless, even in the quenched approximation, there remains an important and hard to quantify systematic error: so far simulation data are obtained at only rather large values of the pseudo scalar mass of $600 \mathrm{MeV}$ of even higher. Clearly, an extrapolation to the physical value, i.e. a pion mass of about $140 \mathrm{MeV}$, is diffi cult since contact to chiral perturbation theory cannot be established with such large pseudo scalar masses.

In fi g. 9 of ref. [8] we give an example of the situation of our earlier calculation of a twist-2, non-singlet operator in pion states, correponding to the average momentum of the parton. The gap between the smallest pseudo scalar mass of about $600 \mathrm{MeV}$ reached in this work and the physical point at $140 \mathrm{MeV}$ appears to be quite large, leaving a substantial uncertainty in confronting the lattice calculations with the experimental results.

In this contribution we will report about a way to overcome the uncertainty of the chiral extrapolation by employing Wilson twisted mass lattice fermions [9, 10] which allows to regulate unphysical, small eigenvalues of the Wilson-Dirac operator, and hence can reach pion masses of well below $300 \mathrm{MeV}$ and thus provide a tool to use chiral perturbation theory to perform the last, small step to extrapolate to the physical point. See [11, 12] for recent reviews.

We emphasize that it is the main goal of this contribution to show that the gap in the pseudo scalar masses, seen in fig. 9 of ref. [\$], can indeed be closed when using Wilson twisted mass fermions. It is not the aim of the paper to provide a phenomenological number of the matrix element under consideration here and to compare to experiment. At the example of a 3-point function, the paper shall provide a demonstration that the Wilson twisted mass approach can be used to simulate small pion masses of $\mathrm{O}(270 \mathrm{MeV})$ while keeping $\mathrm{O}\left(a^{2}\right)$ cut-off effects under control as has been shown already for quantities that derive from 2-point correlation functions [13, 14, 15]. A more detailed account of this work will be published in ref. [16].

\section{Lattice action and operators}

In this paper we will work on a lattice $L^{3} \times T$ with twisted mass fermions the action of which 
can be written as

$$
S[U, \psi, \bar{\psi}]=a^{4} \sum_{x} \bar{\psi}(x)\left(D_{W}+m_{0}+i \mu \gamma_{5} \tau_{3}\right) \psi(x),
$$

with the standard Wilson-Dirac operator $D_{\mathrm{W}}$ and $m_{0}, \mu$ the bare un-twisted and twisted mass parameters, respectively. Here and in the following $\psi(x)$ indicates a flavour doublet of quarks.

In the present work, we will employ two defi nitions for the critical mass with the fi nal aim of a combined continuum extrapolation of the results obtained in the two cases. The fi rst defi nition of the critical mass is the point where the pseudoscalar meson mass vanishes, the second, where the PCAC quark mass vanishes. In the following we will refer to the fi rst situation as the "pion defi nition" and to the second situation as the "PCAC defi nition" of the critical point, as introduced already in [15]. Both defi nitions should lead to $O(a)$-improvement [10, 17, 18, 19], but they can induce very different $O\left(a^{2}\right)$ effects, in particular at small pseudoscalar meson masses [20].

The towers of twist-2 operators related to the unpolarized structure functions have the following expressions

$$
O_{\mu_{1} \cdots \mu_{N}}^{a}(x)=\frac{1}{2^{N-1}} \bar{\psi}(x) \gamma_{\left\{\mu_{1}\right.} \stackrel{\leftrightarrow}{D}_{\mu_{2}} \cdots \stackrel{\leftrightarrow}{D}_{\left.\mu_{N}\right\}} \frac{1}{2} \tau^{a} \psi(x),
$$

where $\{\cdots\}$ means symmetrization on the Lorentz indices, $\stackrel{\leftrightarrow}{D}_{\mu}=\vec{D}_{\mu}-\overleftarrow{D}_{\mu}$ and $\quad D_{\mu}=\frac{1}{2}\left[\nabla_{\mu}+\right.$ $\left.\nabla_{\mu}^{*}\right]$. The flavour structure is specifi ed by the Pauli matrices $\tau^{a}$ where we include here also the identity with $\tau^{4}=2 \cdot \mathbb{1}$. In general one should perform an axial rotation in order to have the expressions for the twist- 2 operators for the twisted mass formulation. However, the operators in eq. (2.2) with flavour index $a=1,4$ do not rotate. We concentrate in this work on the twist-2 quark operator related to the lowest moment of the valence quark parton distribution function in a pion. In particular we will use for the up quark (the down quark can be treated in the same way)

$$
\mathscr{O}_{44}^{u}(x)=\frac{1}{2} \bar{\psi}(x)\left[\gamma_{4} \stackrel{\leftrightarrow}{D}_{4}-\frac{1}{3} \sum_{k=1}^{3} \gamma_{k} \stackrel{\leftrightarrow}{D}_{k}\right] \frac{\left(1+\tau^{3}\right)}{2} \psi(x) .
$$

The matrix elements of this operator can be computed in the standard way, described in refs. [21, 22]. We indicate with $P^{ \pm}(x)=\bar{\psi}(x) \gamma_{5} \frac{\tau^{ \pm}}{2} \psi(x)$ and $\tau^{ \pm}=\frac{\tau^{1} \pm i \tau^{2}}{2}$ the interpolating operator for the charged pion. The ratio of the 2-point and 3-point functions

$$
C_{P}\left(x_{4}\right)=a^{3} \sum_{\mathbf{x}}\left\langle P^{+}(0) P^{-}\left(\mathbf{x}, x_{4}\right)\right\rangle, C_{44}\left(y_{4}\right)=a^{6} \sum_{\mathbf{x}, \mathbf{y}}\left\langle P^{+}(0) \mathscr{O}_{44}\left(\mathbf{y}, y_{4}\right) P^{-}(\mathbf{x}, T / 2)\right\rangle,
$$

is related to the matrix element we are interested in. In particular if we perform a transfer matrix decomposition and we defi ne $R\left(y_{4}\right)=\frac{C_{44}\left(y_{4}\right)}{C_{P}(T / 2)}$, we obtain, in the limit when only the fundamental state dominates $\left(0 \ll y_{4} \ll T / 2\right)$, the relevant bare quantity as

$$
\langle x\rangle^{\text {bare }}=\frac{1}{m_{\pi}} \cdot R .
$$

The matrix element obtained in this way has to be renormalized by a multiplicative renormalization factor. To this end, we took the $Z_{\mathrm{RGI}}$ as computed in ref. [8, 7] using the Wilson action. The renormalized matrix element has a well defi ned continuum limit and in the phenomenological relevant $\overline{\mathrm{MS}}$ scheme is given by

$$
\langle x\rangle^{\overline{\mathrm{MS}}}\left(\mu, r_{0} m_{\pi}\right)=\lim _{a \rightarrow 0} \frac{\langle x\rangle^{\text {bare }}\left(a, m_{\pi}\right) Z_{\mathrm{RGI}}(a)}{f^{\overline{\mathrm{MS}}}(\mu)} \quad \mu=2 \mathrm{GeV},
$$

where $Z_{\mathrm{RGI}}$ and $f^{\overline{\mathrm{MS}}}(\mu)$ were computed in ref. [8] (see this reference for further details). 


\section{Numerical results}

Our quenched simulations were performed for a number of bare quark masses in a corresponding pion mass range of $270 \mathrm{MeV}<m_{\pi}<1.2 \mathrm{GeV}$ using the Wilson plaquette gauge action, employing periodic boundary conditions for all fi elds. In ref. [16] detailed tables with the numerical results can be found. We performed simulations at fi xed values of the twisted mass parameter $\mu$ while keeping $m_{0}$ to be the critical quark mass as obtained from the pion or PCAC defi nitions.

Using the force parameter $r_{0}=0.5 \mathrm{fm}$ to set the scale we determined the physical value of the pion mass and interpolated the values of $\langle x\rangle$ for the chosen values of $m_{\mathrm{PS}} r_{0}$. These values are close to the simulated ones, and so even a linear interpolation is usually suffi cient. The lowest mass that can be reached corresponds to $m_{\mathrm{PS}}=272 \mathrm{MeV}$. Note that we corrected our values for $\langle x\rangle$ for fi nite size effects using the results of ref. [6].

In fig. $]$ we show the continuum extrapolation of $\langle x\rangle$, already converted to the $\overline{\mathrm{MS}}$ scheme as explained in the previous section, at fi xed pion mass for a wide range of pion masses. The scaling of $\langle x\rangle$ is in agreement with the expected $\mathrm{O}\left(a^{2}\right)$ cut-off effects for both defi nitions of the critical mass. The slope of $\langle x\rangle$ as a function of $a^{2}$ appears to be rather small, at least for the pion masses investigated here. It also seems that the slope changes sign from positive to negative values when the pion mass is decreased. The plot also reveals that the continuum extrapolations for $\langle x\rangle$ performed independently for both defi nitions of the critical mass nicely agree.
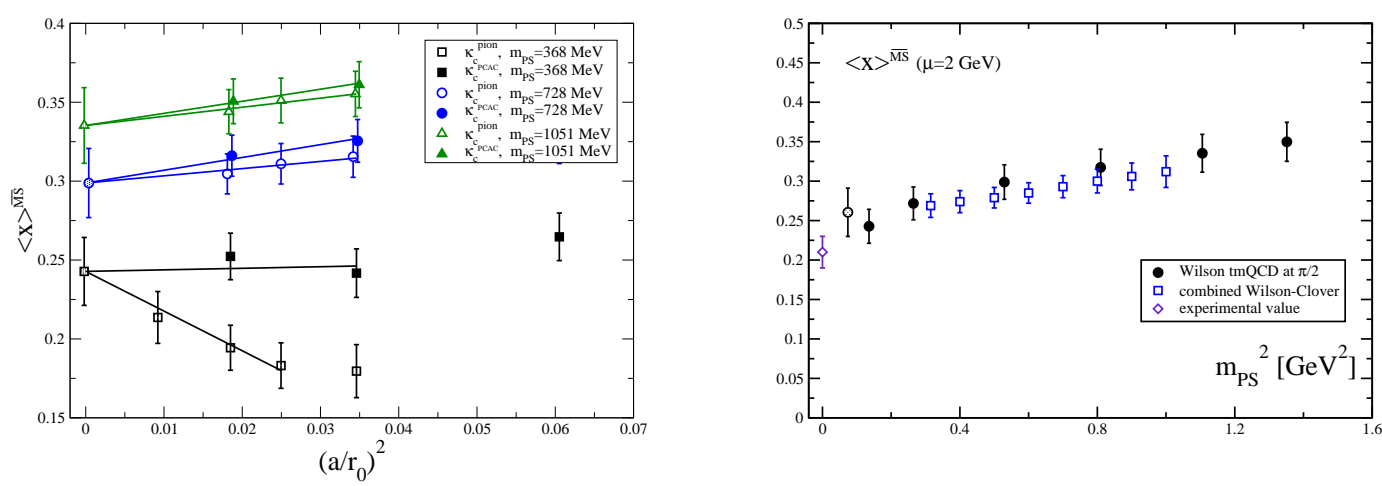

Figure 1: Left panel: $\langle x\rangle^{\overline{\mathrm{MS}}}$ as a function of $a^{2}$ for different values of the pion mass. Right panel: $\langle x\rangle^{\overline{\mathrm{MS}}}(\mu=$ $2 \mathrm{GeV}$ ) extrapolated to the continuum as a function of the pion mass. Open squares represent results that are obtained from a combined continuum extrapolation of earlier Wilson and clover-Wilson simulations, ref. [8]. The filled cicles represent our results using Wilson twisted mass fermions. The open circle denotes a result which is not corrected for finite size effects and the diamond corresponds to the experimental point.

In fig. 1 (right panel) we show $\langle x\rangle^{\overline{\mathrm{MS}}}$ in the continuum as a function of the continuum pion mass. The open squares represent our values obtained earlier from a combined continuum extrapolation of Wilson and clover-improved Wilson fermions using the Schrödinger functional scheme [8]. As usual, such simulations have to stop at a pion mass of about $600 \mathrm{MeV}$. At such high pion masses it becomes very diffi cult, if not impossible to compare the simulation results, even when extrapolated to the continuum limit as done here, to chiral perturbation theory. 
The right panel of Fig. 1 shows that with Wilson twisted mass fermions, represented by the fi lled circles, the large gap between a pion mass of about $600 \mathrm{MeV}$, as the lower bound for standard simulations, and the physical value can be bridged. The Wilson twisted mass results for $\langle x\rangle^{\overline{\mathrm{MS}}}$ are consistent with a linear behaviour. Note that we add in the fi gure a point denoted with an open circle which is not corrected for fi nite size effects and is shown for illustration purpose only that also pion mass below $300 \mathrm{MeV}$ can be reached with Wilson twisted mass fermions.

\section{Conclusions}

In this contribution we have computed the lowest moment of a pion parton distribution funtion. In particular, we concentrated on a twist-2, non-singlet operator the matrix element of which corresponds to the average momentum of a parton in the pion. We demonstrated, that also for such matrix elements pseudo scalar masses below $300 \mathrm{MeV}$ can be reached. At the same time, the cut-off effects even at such small pseudo scalar masses appear only in $\mathrm{O}\left(\mathrm{a}^{2}\right)$ and remain small and controllable. This clearly opens the door to make contact to chiral perturbation theory and to perform reliable extrapolations to the physical point with a pseudo scalar mass that corresponds to the experimentally observed pion mass.

In the present work we have employed two defi nitions of the critical mass, the pion and PCAC mass defi nition [14, 18]. We could perform a controlled continuum extrapolation of $\langle x\rangle$ at least down to pion masses of about $270 \mathrm{MeV}$ by combining the data obtained with the two defi nitions. Of course, in principle, also overlap simulations are able to reach such values of the pion mass. This will come, however, at a much higher simulation cost [23]. The right panel of our fi nal fi gure, fi g.1, clearly demonstrates that with our present setup it is possible to go beyond standard calculations and bridge the gap between large pion mass values of $600 \mathrm{MeV}$ and the physical value of the pion mass.

\section{Acknowledgements}

We thank Giancarlo Rossi and Stefan Sint for many useful discussions. The computer centers at NIC/DESY Zeuthen, NIC at Forschungszentrum Jülich and HLRN provided the necessary technical help and computer resources. S. C. gratefully acknowledges support by Fonds zur Förderung der Wissenschaftlichen Forschung in Österreich, Project P16310-N08. This work was supported by the DFG Sonderforschungsbereich/Transregio SFB/TR9-03.

\section{References}

[1] R. Devenish and A. Cooper-Sarkar, Deep inelastic scattering, . Oxford, UK: Univ. Pr. (2004) 403 p.

[2] J. Blumlein and H. Bottcher, Qcd analysis of polarized deep inelastic scattering data and parton distributions, Nucl. Phys. B636 (2002) 225-263 hep-ph/0203155.

[3] S. Alekhin, Parton distributions from deep-inelastic scattering data, Phys. Rev. D68 (2003) 014002 [hep-ph/0211096].

[4] R. S. Thorne, A. D. Martin, W. J. Stirling and R. G. Roberts, Update of mrst parton distributions, hep-ph/0407311. 
[5] M. Guagnelli, K. Jansen and R. Petronzio, Universal continuum limit of non-perturbative lattice nonsinglet moment evolution, Phys. Lett. B457 (1999) 153-156 hep-lat/9901016.

[6] Zeuthen-Rome (ZeRo) Collaboration, M. Guagnelli et. al., Finite size effects of a pion matrix element, Phys. Lett. B597 (2004) 216-221 hep-lat/0403009.

[7] Zeuthen-Rome (ZeRo) Collaboration, M. Guagnelli et. al., Continuous external momenta in non-perturbative lattice simulations: A computation of renormalization factors, Nucl. Phys. $\mathbf{B 6 6 4}$ (2003) 276-298 [hep-lat/0303012].

[8] Zeuthen-Rome (ZeRo) Collaboration, M. Guagnelli et. al., Non-perturbative pion matrix element of a twist-2 operator from the lattice, Eur. Phys. J. C40 (2005) 69-80 [hep-lat/0 05027 ].

[9] ALPHA Collaboration, R. Frezzotti, P. A. Grassi, S. Sint and P. Weisz, Lattice QCD with a chirally twisted mass term, JHEP 08 (2001) 058 [hep-lat / 0101001 ].

[10] R. Frezzotti and G. C. Rossi, Chirally improving Wilson fermions. I: O(a) improvement, JHEP 08 (2004) 007 hep-lat/0306014.

[11] R. Frezzotti, Twisted mass lattice QCD, Nucl. Phys. Proc. Suppl. 140 (2005) 134-140 [hep-lat/0409138].

[12] A. Shindler, Twisted mass lattice QCD: recent developments and results, . Plenary talk at Lattice 2005.

[13] A. M. Abdel-Rehim, R. Lewis and R. M. Woloshyn, Spectrum of quenched twisted mass lattice QCD at maximal twist, Phys. Rev. D71 (2005) 094505 [hep-lat/0503007.

[14] XLF Collaboration, K. Jansen, M. Papinutto, A. Shindler, C. Urbach and I. Wetzorke, Light quarks with twisted mass fermions, Phys. Lett. B619 (2005) 184-191 [hep-lat/0503031].

[15] XLF Collaboration, K. Jansen, M. Papinutto, A. Shindler, C. Urbach and I. Wetzorke, Quenched scaling of Wilson twisted mass fermions, hep-lat/0507010.

[16] S. Capitani, K. Jansen, M. Papinutto, A. Shindler, C. Urbach and I. Wetzorke, In preparation, .

[17] S. Aoki and O. Bär, Twisted-mass $Q C D, O(a)$ improvement and Wilson chiral perturbation theory, Phys. Rev. D70 (2004) 116011 [hep-lat/0409006].

[18] S. R. Sharpe and J. M. S. Wu, The phase diagram of twisted mass lattice QCD, Phys. Rev. D70 (2004) 094029 [hep-lat/0407025].

[19] S. R. Sharpe and J. M. S. Wu, Twisted mass chiral perturbation theory at next-to-leading order, Phys. Rev. D71 (2005) 074501 [hep-lat/0 411021].

[20] R. Frezzotti, G. Martinelli, M. Papinutto and G. C. Rossi, Reducing cutoff effects in maximally twisted lattice QCD close to the chiral limit, hep-lat/0503034.

[21] G. Martinelli and C. T. Sachrajda, Pion structure functions from lattice QCD, Phys. Lett. B196 (1987) 184.

[22] C. Best et. al., Pion and rho structure functions from lattice QCD, Phys. Rev. D56 (1997) 2743-2754 [hep-lat/9703014].

[23] XLF Collaboration, T. Chiarappa et. al., Comparing iterative methods for overlap and twisted mass fermions, hep-lat/0409107, and in preparation. 